\title{
Sudden Infant Death Syndrome Victims Show Local Immunoglobulin M Response in Tracheal Wall and Immunoglobulin A Response in Duodenal Mucosa
}

\author{
LAURITZ STOLTENBERG, OLA D. SAUGSTAD, AND TORLEIV O. ROGNUM \\ Institute of Forensic Medicine and Department of Pediatric Research, University of Oslo, The National Hospital, \\ Oslo, Norway
}

\begin{abstract}
Twenty-two sudden infant death syndrome (SIDS) cases and 22 controls were examined immunohistochemically with regard to $\operatorname{IgA}$, IgM, and IgG plasma cells in tracheal wall and duodenal mucosa. Furthermore, the presence of secretory component in tracheal surface and gland epithelium as well as in duodenal crypt and villus epithelium were evaluated. The examined specimens were obtained at autopsies. The control groups consisted of 11 infants who died of noninfectious causes and 11 who died of infections. In the tracheal wall, the SIDS group had higher IgM cell numbers than the control group that died of noninfectious causes $(p<0.01)$, whereas the SIDS victims had lower IgA and IgM cell numbers than the infectious control group $(p<0.01)$. In the duodenal mucosa, the SIDS group had significantly higher IgA cell numbers than the noninfectious control group $(p<0.02)$ but lower IgA cell numbers than the infection group $(p<$ 0.01 ). Secretory component was present in the epithelium from all SIDS cases and controls, both in the tracheal wall glands and in the duodenal crypt mucosa. These findings indicate that the mucosal immune system is stimulated in SIDS. (Pediatr Res 31: 372-375, 1992)
\end{abstract}

\section{Abbreviations}

SIDS, sudden infant death syndrome

SC, secretory component

Hx, hypoxanthine

Among the Scandinavian countries, Norway has the highest incidence of SIDS, and the incidence seems to be increasing (1). Both the etiology and the pathogenesis of SIDS are at present unclear. We have, however, recently published data that indicate that hypoxia precedes death in approximately $80 \%$ of the SIDS cases $(2,3)$. There are several factors that might initiate respiratory inhibition and hypoxia. Because SIDS is more common in colder climates and an increased incidence correlates with the season of respiratory tract infections (4), an immune response in the respiratory tract might be one possible "trigger mechanism."

Adaptive immunity at mucosal surfaces of children and adults is mainly exerted by secretory $\operatorname{IgA}$ and $\operatorname{IgM}$ antibodies, which represent the quantitatively most important humoral immune system of the body (5-8). SC, the poly-Ig receptor, is imperative

Received March 27, 1991; accepted November 21, 1991.

Correspondence and reprint requests: Dr. Lauritz Stoltenberg, Institute of Forensic Medicine, University of Oslo, The National Hospital, N-0027 Oslo, Norway.

Supported by the Norwegian Red Cross, Norwegian SIDS Society, Grieg Ltd. and The Norwegian Research Council for Science and the Humanities. for the secretion of secretory IgA and secretory $\operatorname{lgM}(9)$. Increased concentrations of $\operatorname{IgM}, \operatorname{IgG}$, and to a lesser extent IgA in lung lavage fluid from SIDS victims (10) and raised numbers of IgA, $\mathrm{IgM}$, and $\mathrm{IgG}$ producing cells in salivary glands (11) indicate a stimulation of the secretory immune system in the upper airways and lungs. Furthermore, lack of SC in the tracheal epithelium preventing the transport of dimeric $\operatorname{IgA}$ and pentameric IgM has been suggested as an etiologic factor in SIDS (12).

The purpose of this study was to investigate the humoral immune response and the expression of epithelial SC in the wall of the upper respiratory tract and the duodenal mucosa in SIDS.

\section{MATERIALS AND METHODS}

Tissue specimens. Tracheal wall and duodenal mucosa specimens were obtained from 22 infants who died from SIDS (15 males and seven females; median age $4 \mathrm{mo}$, range 1-7 mo). Tracheal wall samples were taken from the carina; one sample was rejected because of bacterial growth.

The control group consisted of 22 specimens from tracheal wall and from duodenal mucosa. The median age of the control group was 4 mo (range $1-12 \mathrm{mo}$ ). Eleven infants died of the following causes: congenital heart disease $(n=4)$, head injury ( $n$ $=2$ ), infanticide $(n=1)$, CO intoxication $(n=1)$, drowning ( $n$ $=1)$, battered child syndrome $(n=1)$, and accidental suffocation $(n=1)$. None of these infants had clinical evidence of an infection. Eleven infants died of a specific infection: sepsis $(n=$ 4 ), pneumonia $(n=6)$, or meningitis $(n=1)$. All tissue specimens were examined independently by two pathologists.

Clinicopathologic data were obtained from birth clinics and local hospitals admitting the SIDS infants.

Immunohistochemistry. Two adjacent tissue samples approximately $1 \mathrm{~mm}$ thick were excised from each specimen. One was fixed directly in cold ethanol $4^{\circ} \mathrm{C}$, whereas the other was extracted

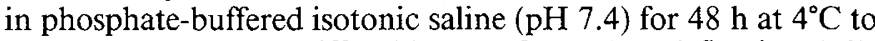
remove extracellular diffusable Ig before ethanol fixation (13). Thereafter, both samples were processed for paraffin embedding. Ig-producing immunocytes. Serial sections cut at $6 \mu \mathrm{m}$ from the PBS-extracted samples were evaluated by paired immunohistochemical staining. Control of the staining specificity and the preparation and characterization of fluorescein isothiocyanate and tetramethylrhodamine isothiocyanate fluorochrome conjugates have been reported elsewhere (14). They were all rabbit reagents with specificities for human $\operatorname{IgA}, \operatorname{IgM}$, and $\operatorname{IgG}$, and were applied in various pairs of contrasting colors. Furthermore, fluorescein isothiocyanate-labeled sheep anti-SC was combined with tetramethylrhodamine isothiocyanate-labeled anti-IgA (15).

Epithelial expression of SC. SC were demonstrated using the following procedure: One section was stained by a routine method using hematoxylin, azophloxin, and saffron (16). The 
adjacent section was first incubated for $30 \mathrm{~min}$ with a tetramethylrhodamine isothiocyanate-labeled sheep IgG-anti SC; its OD ratio $(280 \mathrm{~nm} / 515 \mathrm{~nm})$ was 1.7 , and its working concentration was $0.64 \mathrm{~g} \mathrm{IgG/L} \mathrm{(15).}$

Microscopy, photography, and cell counting. A Leitz Aristoplan (Leica Microscopie und Systeme GmbH, Wetzlar, Germany) microscope was used. It was equipped with a Ploem-type vertical illuminator providing narrow-band excitation and selective filtration of red and green emission colors. Both tracheal and duodenal sections were examined with regard to the presence of IgA, IgM, and IgG plasma cells in the lamina propria and submucosa. We examined the entire cross-section of the carina. An occular grid (Leitz code no. 519902) was applied. The grid area was $0.01 \mathrm{~mm}^{2}$. In most specimens both branches of the bronchus were intact, while in some, one bronchus was partly destroyed. In the duodenal samples, areas with preserved histologic structures were examined. Density of plasma cells was given as cell number per grid area. All fluorescent cells with a discernible nucleus and cell-like body with a pure green or red cytoplasmic color were included.

Cell enumeration and reproducibility of procedure. To obtain a representative mean cell count for each subject, it was necessary to test the number of grids that had to be counted to get a stable mean.

The cell counting was performed independently by two different observers (L.S. and T.O.R.) in 22 grid areas, and the results were subjected to correlation analyses. The ability to reproduce the whole procedure-sectioning, staining, and cell enumeration by the same observer-was tested blindly after 5 mo on 28 specimens.

Epithelial expression of SC was either judged to be present or not present. Tracheal gland epithelium and surface epithelium, as well as duodenal crypt epithelium and villous epithelium, were examined separately.

Clinical variables. Birth characteristics, weight gain, time of the year, history of respiratory tract infections, microbiologic findings, as well as pathologic findings at the autopsy, especially lung microscopy, were recorded.

Corpus vitreum $H x$ levels. Corpus vitreum $\mathrm{Hx}$ levels were determined by HPLC $(2,3)$ and corrected according to postmortem time (3).

Statistical analysis. The two-tailed Mann-Whitney U test was used for comparison between the two groups. For testing of interpersonal reproducibility of cell counting and for reproducibility of the whole procedure, the Pearson/correlation test was applied.

\section{RESULTS}

Representative cell enumeration. To obtain a stable mean for each subject investigated, it was necessary to count 20 grids in each section.

Reproducibility. Cell counts by two different observers were significantly correlated $(r=0.97, p<0.001)$. The blind reproducibility test of the staining procedure and cell enumeration by the same observer was also significantly correlated $(r=0.98, p$ $<0.001)$

Ig-producing cells. The tracheal lamina propria and submucosa of SIDS victims contained significantly $(p<0.01)$ more IgM plasma cells (median 2.3; range 1.6-4.0) than controls (median 0.95 ; range $0.8-1.5$ ). For the IgA and IgG isotypes, no difference was found. The infectious control group had significantly $(p<$ 0.01 ) more IgA plasma cells (median 9.4; range 6.2-13.4) than the SIDS group (median 5.0; range 3.4-7.8) and more IgM plasma cells (median 4.9; range 1.4-6.1) than the SIDS group (median 2.3; range 1.6-4.0) (Fig. 1). Duodenal mucosa from SIDS cases contained significantly $(p<0.02)$ more IgA plasma cells (median 7.0; range 4.4-11.2) than controls (median 5.0; range 3.4-7.4) (Fig. 2A). No such difference was seen for the isotypes IgM and IgG. The infectious control group had signifi-

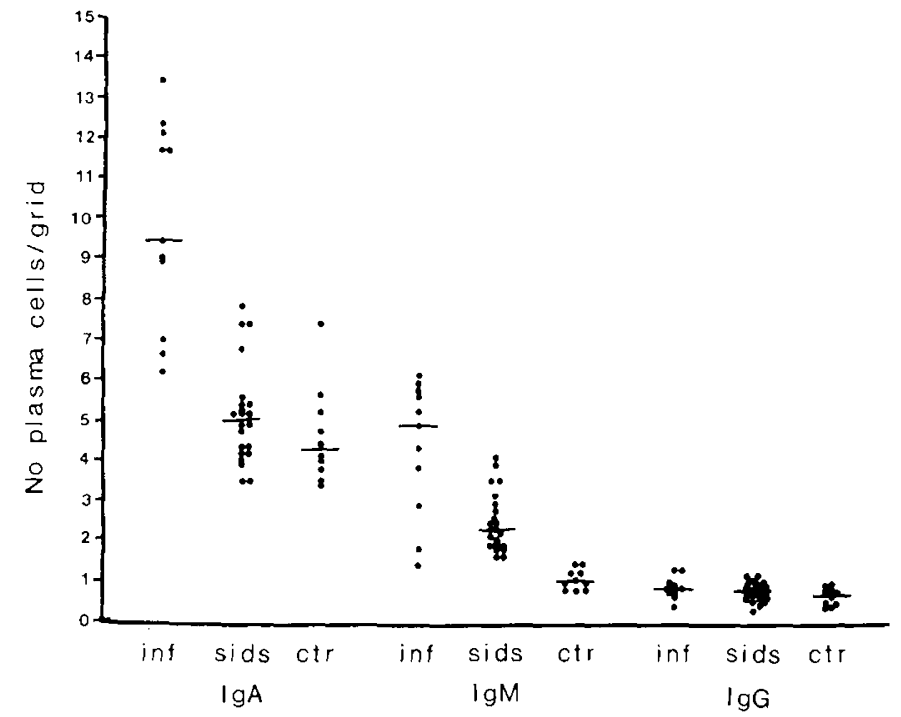

Fig. 1. Number of Ig-producing cells in the tracheal wall of subjects with SIDS (sids) and controls (ctr). SIDS tracheal lamina propria and submucosa contain significantly $(p<0.01)$ more IgM plasma cells than controls. The infectious control group (inf) contains significantly $(p<$ 0.01 ) more IgA and IgM than the subjects with SIDS.

cantly $(p<0.01)$ more IgA plasma cells (median 12 ; range 6.818.8) than the SIDS group (median 7.0; range 4.4-11.2) (Fig. 3).

Epithelial SC. Epithelial SC was present in tracheal wall glands and duodenal crypt mucosa in all SIDS cases and all controls (Fig. $2 B$ ), whereas the expression of this marker was sometimes absent in the tracheal surface epithelium and always very weak in duodenal villi epithelium of both groups.

Clinical variables. Eighteen of the infants were born at term (38-42 wk of gestation), whereas four were premature and one was postmature (median $394 / 7 \mathrm{wk}$; range 33 to $42^{3 / 7} \mathrm{wk}$ ). Ten of the SIDS victims had relatively poor weight gain, i.e. $<50 \%$ of expected weight gain according to birth weight, length, and head circumference (17).

Twenty infants (90\%) died in the period between October and April. Thirteen infants (59\%) had definite signs and symptoms of an upper respiratory tract infection, rhinitis, cough, and/or fever. Nose swabs from two of these infants gave growth to Haemophilus influenzae; one nasopharyngeal aspirate was positive on adenovirus and another one was positive on respiratory syncytial virus. In eight of the infants with clinical symptoms of an upper respiratory infection, lung microscopy showed signs of inflammation, slight thickening of alveolar septa, and slight increase in lymphocytes and macrophages around segmental bronchi. These changes were judged to be insufficient to cause death.

Corpus vitreum $H x$ levels. Eighteen of 20 investigated SIDS subjects had increased vitreous humor Hx levels when compared with 11 noninfectious and 11 infectious controls $(p<0.01)$ after the values had been corrected for expected postmortem time increase. The results are given in Table 1 and are part of a previous study (3).

\section{DISCUSSION}

The presence of SC in tracheal and duodenal epithelium is in contrast to the theory put forward by Ogra et al. (12) in 1975 that lack of SC might represent an etiologic factor in SIDS. The most important finding of the present investigation, however, was the increased number of IgM immunocytes in the tracheal wall and of IgA immunocytes in duodenal mucosa in SIDS victims. One great problem with studies in SIDS is to have sufficient normal controls. To our knowledge, there is only one previous study on the humoral mucosal immune system of the 

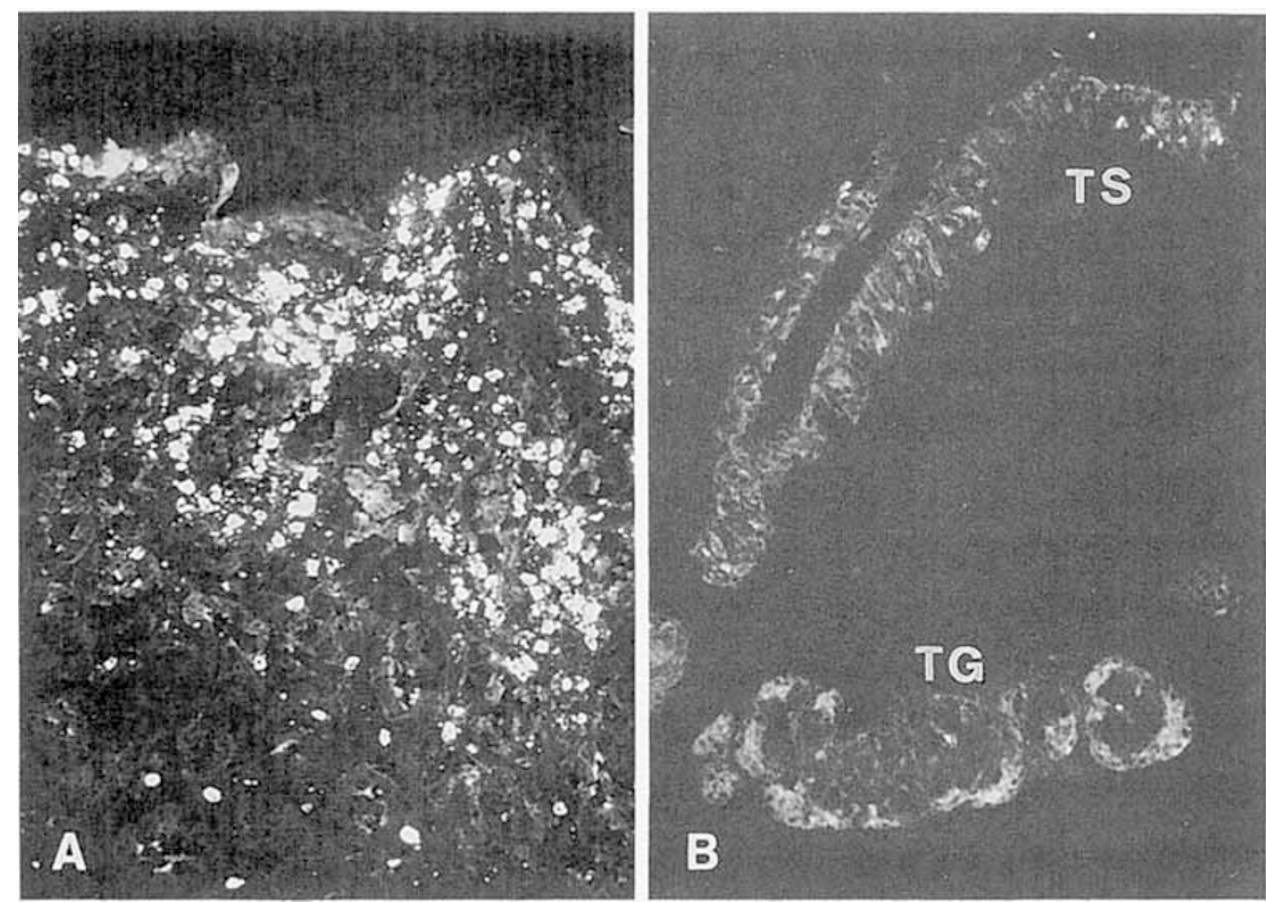

Fig. 2. A, Immunofluorescence staining of $\operatorname{IgA}$ in duodenal mucosa from a case of SIDS. Numerous IgA immunocytes are present, and the crypt epithelium is intensely IgA-positive. Magnification $\times 90 . B, \mathrm{SC}$ in tracheal wall in SIDS. SC is present in both tracheal gland epithelium $(T G)$ and in tracheal surface epithelium $(T S)$. Magnification $\times 90$.

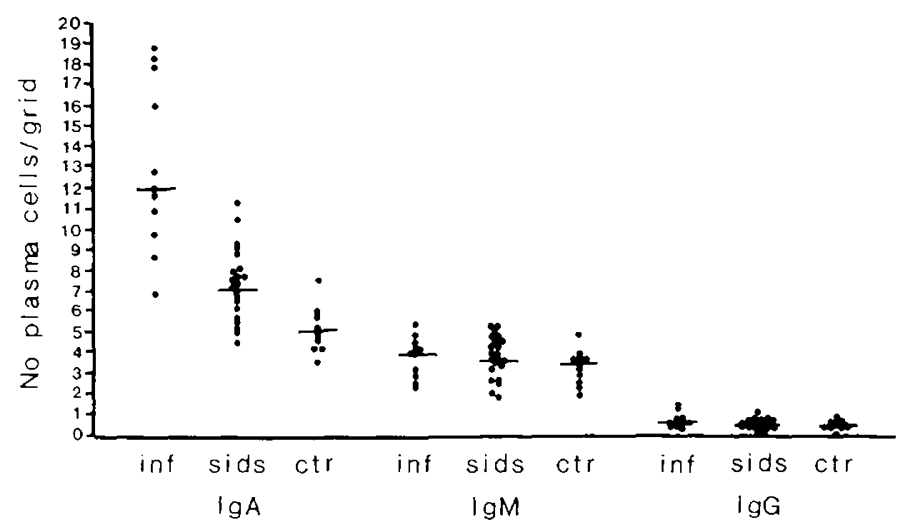

Fig. 3. Number of Ig-producing cells in the duodenal mucosa from subjects with SIDS (sids) and controls (ctr). SIDS duodenal mucosa contains significantly $(p<0.02)$ more IgA plasma cells than controls. The infectious control group (inf) contains significantly $(p<0.01)$ more IgA than the SIDS group.

Table 1. Hx levels in SIDS cases and controls ( $\mu \mathrm{mol} / L)^{*}$

\begin{tabular}{ccc}
\hline & Median & Range \\
\hline SIDS $(n=22)$ & 253 & $39-603$ \\
Control $(n=11)$ & 18 & $4-32$ \\
Infection $(n=11)$ & 64 & $6-148$ \\
\hline
\end{tabular}

* The values are corrected for postmortem time.

respiratory tree (18). This study on segmental bronchi included nine infants from the time of birth to the age of $1 \mathrm{y}$. The authors found ratios of $\mathrm{IgA}$, IgM, and IgG immunocytes that were comparable to our observations in the trachea. Additionally, our own recent study on the ontogeny of tracheal mucosal immunity gave the same results (Stoltenberg L, Thrane PS, Rognum TO, unpublished observations). The density of $\operatorname{IgA}$, IgM, and IgG immunocytes in duodenal mucosa in the normal controls in the present study was similar to that observed in a previous investigation (18a).
The increased number of IgM immunocytes in SIDS tracheal lamina propria and submucosa, as well as the IgA response in SIDS duodenal mucosa indicates that both lymphoid tissue in the upper respiratory tract and gut-associated lymphoid tissue are stimulated in SIDS. These findings are in agreement with those of Forsyth et al. (10) on lung lavage fluid and Thrane et al. (11) in salivary glands. The cause of this stimulation is not known. It is, however, possible that a viral infection in the upper respiratory tract may elicit such an immune response (19). The fact that $13(59 \%)$ of our SIDS population had definite clinical signs and symptoms of an upper respiratory tract infection before death supports this suggestion. It is well known that SIDS is more frequent during epidemics of respiratory syncytial virus and whooping cough (20).

Eight of those with symptoms $(61.5 \%)$ had IgM cell numbers above the median in their carina specimens, whereas $11(85 \%)$ had IgA cell numbers above the median in their duodenal specimens, indicating a strong relationship between the high density of these immunocyte isotypes and respiratory infections. However, the group with lethal infections had significantly higher immunocyte numbers than the SIDS infants. This difference might indicate that the immune response demonstrated in the SIDS group is by itself not lethal. The fact that the infectious control infants had significantly lower $(p<0.02) \mathrm{Hx}$ levels in their vitreous humor than the SIDS infants suggests that the death mechanism in the former group is a different one than that in SIDS. However, other potentially predisposing factors such as astrogliosis in the brainstem $(21-23)$ and delayed maturation of the CNS (24) have to be taken into consideration.

Brainstem astrogliosis in SIDS may indicate earlier episodes of hypoxia (20-22). Furthermore, the increased levels of $\mathrm{Hx}$ in the vitreous humor in 18 of the 20 investigated SIDS cases indicate that hypoxia probably was the ultimate death mechanism in these cases, but not in the noninfectious controls (Table 1). The $\mathrm{Hx}$ values in the infectious control group show an intermediate distribution, indicating that hypoxia may play a role in some of these cases. Other death mechanisms such as sudden cardiac arrest or ventricular fibrilation $(25,26)$, as well as metabolic disorders (27), must be considered in the two infants 
with normal $\mathrm{Hx}$ values who had SIDS (two infants were not investigated). These findings support our belief that some infants are probably "at risk" already at birth and that these infants are particularly vulnerable at the age of 2-4 mo, as suggested by other researchers $(23,28)$.

A possible cause of autonomous dysregulation is the delayed maturation of the CNS (24), and a possible trigger mechanism for the down-regulation of respiration might be an immune response in the periphery communicated to the CNS by bloodborne cytokines or perhaps by retrograde axonal signals (29). The immune response demonstrated in the present study may thus act as a trigger mechanism for the fatal hypoxia in SIDS (2, $3)$. Furthermore, immune responses in organs other than those investigated in this study may act in a similar fashion (11).

A prolonged sleep apnea occuring in a predisposed child with inhibited arousal may lead to severe hypoxia, coma, and death. More studies involving measurements of various cytokines in cerebrospinal fluid and brain tissue from SIDS cases and controls as well as animal experiments may offer a better understanding of the trigger mechanisms in SIDS.

Acknowledgments. The authors thank Gunn Jamne at the Institute of Pathology, Laboratory for Immunohistochemistry and Immunopathology (LIIPAT) and Hanne Malmstrøm for technical assistance.

\section{REFERENCES}

I. Irgens LM, Skjaerven R, Lie RT 1989 Secular trends of sudden infant death syndrome and other causes of post perinatal mortality in Norwegian birth cohorts. Acta Paediatr Scand 78:228-232

2. Rognum TO, Saugstad OD, Oyasaeter S, Olaisen B 1988 Elevated levels of hypoxanthine in vitrous humor indicate prolonged cerebral hypoxia in victims of sudden infant death syndrome. Pediatrics 82:615-617

3. Rognum TO, Saugstad OD 1991 Hypoxanthine levels in vitrous humor. Evidence of hypoxia in most cases of SIDS. Pediatrics 87:306-310

4. Uren EC, Williams AL, Jack I, Rees JW 1980 Association of respiratory virus infection with sudden infant death syndrome. Med J Aust 1:417-419

5. Brandtzaeg P 1983 The secretory immune system of lactating human mammary glands compared with other exocrine organs. Ann NY Acad Sci 409:353-381

6. Brandtzaeg P 1989 Salivary immunoglobulins. In: Tenuovuo (ed) Human Saliva: Clinical Chemistry and Microbiology, Vol. II. CRC Press, Boca Raton, FL, pp $1-54$

7. Brandtzaeg P, Baklien K, Bjerke K, Rognum TO, Scott H, Valnes K 1987 Nature and properties of the human gastrointestinal immune system. In: Miller K, Nicklin S (eds) Immunology of the Gastrointestinal Tract. CRC Press, Boca Raton, FL, pp 1-86

8. Brandtzaeg P, Halstensen TS, Kett K, Krajci P, Kvale D, Rognum TO, Scot H, Sollid LM 1989 Immunobiology and immunopathology of human gut mucosa: humoral immunity and intraepithelial lymphocytes. Gastroenterology 97:1562-1584

9. Brandtzaeg P 1985 Role of $J$ chain and secretory component in receptormediated glandular and hepatic transport of immunoglobulins in man. Scand J Immunol 22:111-146

10. Forsyth KD, Weeks SC, Skinner J, Bradley J 1989 Lung immunoglobulins in the sudden infant death syndrome. Br Med J 286:23-26

11. Thrane PS, Rognum TO, Brandtzaeg P 1990 Sudden infant death syndrome (SIDS): increased immune response in upper respiratory and digestive tracts in SIDS. Lancet 335:229-330

12. Ogra PL, Ogra SS, Coppola PR 1975 Secretory component in sudden infant death syndrome. Lancet 28:387-390

13. Brandzaeg P 1974 Mucosal and glandular distribution of immunoglobulin components. Immunohistochemistry with cold ethanol-fixation techniques. Immunology 26:1101-1114

14. Baklien K, Brandtzaeg P 1975 Comparative mapping of the local distribution of immunoglobulin containing cells in ulcerative colitis and Crohn's disease of the colon. Clin Exp Immunol 22:197-209

15. Rognum TO, Brandtzaeg P, Oerjasaeter H, Elgjo K, Hognestad J 1980 Immunohistochemical study of secretory component, secretory IgA and carcinomembryonic antigen in large bowel carsinoma. Pathol Res Pract 170:126145

16. Stave R, Brandtzaeg P 1977 Fluorescence staining of gastric mucosa. A study with special reference to parietal cells. Scand J Gastroenterol 12:885-891

17. Knudtzon J, Waaler PE, Skjaerven R, Solberg LK, Steen J 1988 Height, weight and head circumference in 0-4 year old infants. Databased registration from the medical birth register. Tidsskr Nor Laegeforen 26:2136-2142

18. Takemura T, Eishi Y 1985 Distribution of secretory component and immunoglobulins in the developing lung. Am Rev Respir Dis 131:125-130

18a. Rognum TO, Thrane PS, Stoltenberg L, Vege A, Brandtzaeg P 1992 Development of intestinal immunity in fetal life and the first postnatal months. Pediatr Res (in press)

19. Minami A, Fujimoto K, Ozaki Y, Nakamura S 1988 Augmentation of host resistance to microbial infections by recombinant human interleukin- 1 alpha. Infect Immun 56:3116-3120

20. Nicoll A, Gardner A 1988 Whooping cough and unrecognized postperinatal mortality. Arch Dis Child 63:4 I-47

21. Naeye RL 1980 Sudden infant death. Sci Am 242:52-62

22. Takashima S, Armstrong D, Becker L, Bryan C 1978 Cerebral hypoperfusion in the sudden infant death syndrome? Brainstem gliosis and vasculature. Ann Neurol 3:257-262

23. Valdes-Dapena M 1986 Sudden infant death syndrome. Morphology update for forensic pathologists-1985. Forensic Sci Int 30:177-186

24. Takashima S, Mito T, Becker LE 1985 Neuronal development in the medullary reticular formation in sudden infant death syndrome and premature infants. Neuropediatrics 16:76-79

25. Krongrad E, O'Neill L 1986 Near miss sudden infant death syndrome episodes? A clinical and electrocardiographic correlation. Pediatrics 6:811-815

26. Bharati S, Krongrad E, Lev M 1985 Study of the conduction system in a population of patients with sudden infant death syndrome. Pediatr Cardiol 6:29-40

27. Emery JL, Howat AJ, Variend S, Vawter GF 1988 Investigation of inborn errors of metabolism in unexpected infant death. Lancet 332:29-31

28. Little RE, Peters DR 1990 SIDS: a review and update. Epidemiol Rev 12:241246

29. Maehlen J, Olsson T, Zachau A, Klareskog L, Kristensson K 1989 Local enhancement of major histocompatibility complex (MHC) class I and II expression and cell infiltration in experimental allergic encephalomyelitis around axotomized motor neurons. J Neuroimmunol 23:125-132 